\title{
DAS STAATSANGEHÖRIGKEITSRECHT DER AMERIKANISCHEN STAATEN
}

\author{
Von Hellmuth Hecker
}

In der folgenden Übersicht wird das Staatsangehörigkeitsrecht der 26 Staaten des amerikanischen Kontinents behandelt. Ein Überblick über die abhängigen Gebiete in Amerika folgt.

Bei der hier abgedruckten Übersicht konnte in vielen Fällen entweder auf Bände der SGS oder auf Überblicke im "Standesamt" (StAZ) zurückgegriffen werden, und zwar sind behandelt:

1.) 8 Staaten in SGS-Bänden: Bolivien, Brasilien, Chile, Columbien, Ecuador, Peru, Venezuela, Vereinigte Staaten

2.) 13 Staaten in der StAZ: Argentinien, Barbados, Costarica, Cuba, Guatemala, Guayana, Honduras, Jamaica, Nicaragua, Panama, Salvador, Trinidad/Tobago, Uruguay

3.) 5 Staaten noch nirgends: Canada, Dominik. Rep., Haiti, Mexiko, Paraguay.

Die meisten der 26 amerikanischen Staaten, nämlich 20, gehören dem lateinischen Rechtskreis an, unter diesen wiederum überwiegt die Beziehung zu der früheren Kolonialmacht Spanien in Sprache, Kultur und Recht. Nur Brasilien und Haiti gehören dem portugiesischen bzw. französischen Kulturbereich an. Sechs Staaten sind abhängig vom Rechtskreis des britischen Common law, nämlich: Barbados, Canada, Guayana, Jamaica, Trinidad und die Vereinigten Staaten.

Die lateinamerikanischen Staaten sind alle der ursprünglichen Praxis der französischen Revolution treu geblieben, wonach die StA dem öffentlichen Recht angehört und in der Verfassung geregelt wird. So finden sich in den meisten lateinamerikanischen Staaten noch heute die grundlegenden Vorschriften über Erwerb und Verlust der StA in den Verfassungen. Da die Verfassungen in den lateinamerikanischen Staaten aber nicht immer zu den stabilsten Rechtsvorschriften gehören, so wechselt auch das StA-Recht ebenso oft wie die Verfassungen. Nur wenige Staaten sind der späteren Rechtstechnik Frankreichs gefolgt, das im Code Civil von 1804 die StA dem bürgerlichen Recht zuordnete und dort regelte. Manchmal gab es auch Widersprüche zwischen Verfassung und Codigo Civil, wie z. B. in Venezuela. Ebenso zögernd folgten diese Staaten der dritten Etappe des französischen StA-Rechts, das ab 1927 in einem gesonderten StA-Gesetz geregelt wurde und wieder als dem öffentlichen Recht zugehörig betrachtet wurde. Nur wenige lateinamerikanische Staaten besitzen ein eigenes StAG, manche nur ein Einbürgerungsgesetz.

Von den sechs anglo-amerikanischen Staaten, die alle einmal britische Kolonien gewesen waren, regeln die vier Neustaaten die StA sowohl in der Verfassung wie in einem StAG, während bei Canada und den USA komplizierte Sonderverhältnisse vorliegen.

Für die Texte der zahllosen lateinamerikanischen Verfassungen konnte auf Teil III des Verfassungsregisters, Ffm 1958, 198 S., verwiesen werden sowie auf die Fortführung von Kehden dazu in VRÜ 1968, S. 196-224.

Zur allgemeinen Frage des südamerikanischen StA-Rechts vergleiche die von der Forschungsstelle für Völkerrecht veröffentlichte Monographie:

P. Moosmayer, Der Gebietsgrundsatz im StAR (ius soli) unter besonderer Berücksichtigung der südamerikanischen Staaten, Ffm 1963, 182 S. (Bd. 9 der Abhandlungen der Forschungsstelle) 
Auf dem Gebiete völkerrechtlicher Verträge zum StAR besteht eine Besonderheit in multilateralen Abkommen, die auf den amerikanischen Raum beschränkt sind, nämlich zwei Abkommen von 1933 (Frauen; allg. StA) und eines von 1906 (Einbürgerung). Dagegen sind nur wenige amerikanische Staaten an den Haager StAVerträgen von 1930 beteiligt (Brasilien, Canada, Columbien, Cuba, Salvador, USA, Chile) oder an dem UNO-Abkommen über die StA der Ehefrau von 1957 (Argentinien, Canada, Cuba, Domin. Rep., Ecuador, Guatemala, Jamaica) oder am Wiener StA-Protokoll von 1961 (Argentinien, Dom. Rep., Panama).

Im Bereich der bilateralen StA-Verträge stehen diejenigen mit den USA und mit Spanien im Vordergrund. Zu letzteren Verträgen, die die Doppelstaatigkeit gegenüber ehemaligen Kolonien Spaniens regeln (Bolivien, Chile, Costarica, Ecuador, Guatemala, Honduras, Nicaragua, Paraguay, Peru), siehe die ausführliche Studie

A. Miaja de la Muela, Los Convenios de doble nacionalidad entre España y algunas Republicas americanas in: Rev. Española de Der. Int. 1966, S. 381-410.

\section{ARGENTINIEN}

Übersicht über Verfassungen (1812-1949), Gesetze (1819-1957),

Verträge (1863-1933) und Schrifttum - in: StAZ 1961, S. 54-57

Verträge

29. 1.1957

18. 4.1961

28. 11.1962

8. 5.1963

11. 6.1963

10. 10.1966

\section{UN-Konvention betr. StA der Ehefrau}

gebilligt von Argentinien mit Vorbehalt: Dekret Nr. 3469 vom 26. 4. 1961 (B. O. vom 13. 5. 61; ADLA 1961, S. 527) bestätigt durch Dekretgesetz vom 13. 9.1963 (s. u.), Unterzeichnung 10. 10. 1963, Inkrafttreten für Argentinien: 8. 1. 1964 (UNTS 479, 370). Wiener StA-Protokoll unterzeichnet von Argentinien am 25. 10.61 Inkrafttreten für Argentinien: 10. 11. 1963 Gebilligt durch Art. 5 des Dekretgesetzes Nr. 7672 vom 13.9.1963 (B. O. vom 19.9.63; ADLA 1963, S. 1759 spanischer Text)

Abkommen mit Dänemark über Wehrdienst von Doppelstaatern

Inkrafttreten: 28.11 .1962

Amtlicher Text: UNTS Bd. 455, S. 429

Abkommen mit Finnland über Wehrdienst von Doppelstaatern

Inkrafttreten: 5. 11. 1963

Gebilligt durch Art. 19 des Dekretgesetzes Nr. 7672 (s. o.) Amtlicher Text: UNTS Bd. 482, S. 309

Spanischer Text: ADLA 1963, S. 1783

Ablıommen mit Belgien über Wehrdienst von Doppelstaatern

gebilligt durch Art. 20 des Dekretgesetzes Nr. 7672 (s. o.) Konkordat mit dem Vatikan

StAB: Art. 5II (Erleichterte Einbürgerung für Geistliche) gebilligt durch Gesetz Nr. 17032 vom 23.11. 1966

Amtlicher Text: B. O. vom 22. 12. 1966

Spanischer Text: ADLA 1966, S. 1606 


\section{Gesetze}

A. Nachträge zur Liste in StAZ 1961, S. 54

28. (30.) 6. 1910

Gesetz Nr. 7029 betr. „Defensa Social“

(Einwanderung)

StAB: Art. 6 (Einbürgerungsverbot für illegale Einwanderer. Einbürgerung wird eingezogen, wenn unter Verletzung dieses Gesetzes erlangt)

Dies Gesetz wurde aufgehoben durch das Strafgesetzbuch (Gesetz Nr. 11179 vom 29.10.1921)

Spanischer Text: ADLA 1889-1919, S. 787

30. 9. (21. 10.) 1926 Wehrgesetz, Nr. 11386

Da Rocha, Leyes Nacionales Clasificadas Bd. 9, 1937, S. 251

StAB: Art. 22 (Verlust der Einbürgerung bei Nichtmeldung, keine Wiedereinbürgerung erlaubt). Der Verlust wurde, da Art. 41 bei Amnestien Rückgängigmachung gestattet, durch die Amnestiegesetze Nr. 11657 vom 30. 9. 1932, Nr. 12348 vom 2. (5.) 2. 1937 (Ley 6, Leg. S. 48) und Nr. 12882 vom 8.11. (1.12.) 1946 (ADLA 1946, S. XXII) ex tunc aufgehoben.

Das frühere Wehrgesetz Nr. 8129 vom 14.7.1911 enthielt keine StAB.

Spanischer Text: ADLA 1920-1940, S. 208, Da Rocha, a. a. O., Bd. 2, 1935, S. 380

17. (30.) 12. 1936 Gesetz Nr.12331 betr. Verhütung von Geschlechtskrankheiten

StAB: Art. 17 Satz 3 (Einbürgerung verloren bei Verurteilung). Durch Dekret Nr. 10638 vom 28. 4. 1944 (B. O. vom 5. 5., ADLA 1944, S. 267) wurden Art. 15 und 17 des Gesetzes geändert, aber nicht obiger Satz 3

Spanischer Text: ADLA 1920-1940, S. 703

Leyes Nacionales Nr. 12295-12356, Buenos Aires 1937, S. 297

Entwurf eines neuen argentinischen StA-Gesetzes vorgelegt vom Instituto de Derecho Internacional in 40 Artikeln

Spanischer Text: Ley 1938, Bd. 10, Leg. S. 64-69

15. 1.1945

Dekret Nr. 536 betr. Staatsverbrechen

StAB: Art. 42III (Einbürgerung verloren bei Verurteilung) Amtlicher Text: B. O. vom 29.1. 1945

Spanischer Text: ADLA 1945, S. 128

6. (15.) 2. 1947

Gesetz Nr. 12951 über den Auswärtigen Dienst

StAB: Art. 68 (im Ausland Geborene gelten als gebürtige Argentinier, nicht als Optanten), dazu: L 57, 713 (CFBA vom 1. 12. 1949)

Amtlicher Text: B. O. vom 5. 3. 1947

Spanischer Text: ADLA 1947, S. 204

20. 10. 1947

Dekret Nr. 32537 betr. Verfahren der Einbürgerung ausländischer Frauen argentinischer Diplomaten

Amtlicher Text: B. O. vom 25. 10. 1947

Spanischer Text: ADLA 1947, S. 841 
27. 9. (12. 10.) 1950 Gesetz Nr. 13985 betr. Staatsverbrechen

StAB: Art. 13III = Art. 42III von 1945 (s. o.)

Amtlicher Text: B. O. vom 16. 10. 1950

Spanischer Text: ADLA 1950, S. 185

6. 7.1951 Gesetz Nr.14031 betr. Aberkennung der StA für

Walter Beveraggi Allende

Amtlicher Text: B. O. vom 11. 7. 1951

Spanischer Text: ADLA 1951, S. 106

B. Nach 1957 erlassene Vorschriften

30. 1.1963

Dekretgesetz Nr. 788 betr. Staatsverbrechen

StAB: Art. 42 Satz $3=$ Art. 42 III von 1945

Amtlicher Text: B. O. vom 2. 2. 1963

Spanischer Text: ADLA 1963, S. 58

24. 5.1963

Dekretgesetz Nr. 4214 betr. Verbot des Kommunismus

StAB: Art. 10 Z. 2 (Verlust der StA Eingebürgerter, die wegen kommunistischer Tätigkeit verurteilt wurden)

Amtlicher Text: B. O. vom 30. 5. 1963

Spanischer Text: ADLA 1963, S. 827

29. 10. (25. 11.) 1964 Gesetz Nr. 16569 betr. StA von Kindern von Argentiniern, die während des Exils ihrer Eltern im Ausland geboren wurden

Amtlicher Text: B. O. vom 3. 12. 1964

Spanischer Text: ADLA 1964, S. 2052 mit Begründung

25. 6.1965

Dekret Nr. 5056 betr. Verfahren der Einbürgerung ausländischer Frauen argentinischer Diplomaten

11 Artikel

Amtlicher Text: B. O. vom 5. 7. 1965

Spanischer Text: ADLA 1965, S. 1432

30. 10. (23. 11.) 1965 Gesetz Nr. 16801 betr. Änderung von Art. 10-11

des Staatsangehörigkeitsgesetzes von 1869

Amtlicher Text: B. O. vom 3.11. 1965

Spanischer Text: ADLA 1965, S. 2169

5. 9. 1966

Ministerielle Resolucion (Ed. y. J.) Nr. 356

betr. Nachweise für Einbürgerung

Amtlicher Text: B. O. vom 14. 9. 1966

Spanischer Text: ADLA 1966, S. 913

10. 11.1966

Gesetz Nr. 17014 betr. Schaffung eines Nationalregisters und eines für Einbürgerungsurkunden

Amtlicher Text: B. O. vom 18.11.1966

Spanischer Text: ADLA 1966, S. 1539

29. 2. 1968

Gesetz Nr. 17671 betr. das Nationalregister

StAB: Art. 37 (Eingebürgerte verlieren StA bei Versäumen der Registrierung ohne Wiedererwerbsmöglichkeit)

Amtlicher Text: B. O. vom 12. 3. 1968

Spanischer Text: ADLA 1968, S. 217

29. 3.1968

Gesetz Nr. 17692 betr. StA von Kindern von Argentiniern, die in internationalen Organisationen tätig sind, nach Art. 68 des Gesetzes 12951 von 1947

Amtlicher Text: B. O. vom 4. 4. 1968

Spanischer Text: ADLA 1968, S. 233 mit Begründung 


\section{Rechtsprechung}

Argentinien als bedeutendes Einwanderungsland weist, ähnlich wie die USA, eine reichhaltige Rechtsprechung zum StA-Recht auf. Im folgenden sind die wichtigsten Urteile des Obersten Gerichtshofs der letzten 40 Jahre verzeichnet, wobei insbesondere reine Verfahrensfragen unberücksichtigt blieben. Einige Urteile unterer Gerichte sind als Parallelen erwähnt. An den Fundstellen sind die Urteile der Vorinstanz sowie dissenting opinions und anderes Material mit abgedruckt. Die Leitsätze aller StA-Urteile des O. G. bis 1956 sind verzeichnet in:

Rodriguez Araya, El derecho internacional interpretado por la Corte

Suprema de la Nacion (1863-1956), Rosario 1958, 284 S.

Die Abkürzungen bedeuten, bei Periodica je Jahrgang und Seite,

F: $\quad$ Fallos de la Corte Suprema (danach zitiert obiges Werk)

J: Jurisprudencia Argentina (ab 1918)

L: $\quad$ La Ley (ab 1936)

GdF: $\quad$ Gaceta del Foro (ab 1916)

CF: Urteil eines Oberlandesgerichts (Camera Federal), insbesondere Buenos Aires (BA)

3. 8. 1928 F $152,95=\mathrm{J} \mathrm{28,} 6$

Bei Verlust der StA durch Wehrvergehen (Art. 22 Ley 11386) Wiedererwerb kraft Amnestie gemäß Art. 41 Ley 11386

Dito: 19. 9. 27 ( J 25, 1200), 7. 11. 27 ( ( 26, 430), 1. 2. 28 ( J 27, 5), 25. 8. 33 (F 169, 8), 12. 7. 37 (F 178, 157), CF Rosario 22. 4.42 (L 26, 561), 17. 9. 48 (F 211, 1670), 6. 12. 48 (F 212, 390)

14. $12.1928 \quad \mathrm{~F} 153,286=\mathrm{J} 28,978$

Stillschweigender Verzirch auf die Einbürgerung liegt vor bei respektlosen Außerungen gegen Argentinien

15. 5. 1929 F $154,283=\mathrm{J} 29,591$

Verliert Eingebürgerter seine argent. StA wegen Wehrvergehen, so erstreckt sich das nicht auf eingebürgerte Ehefrau

18. 4. 1932 F 168,374

Einbürgerung bei Betrug über Moral widerrufbar dito: 27. 6. 1934 (F 170, 429)

10. 8. 1934 F $171,103=\mathrm{L} 4,569$

Einbürgerung widerrufbar bei kommunistischer Agitation

dito: 15. 6. 38 (F 181, 54), 26. 7. 39 (L 15, 461), CF BA 12. 3. 41 (L 23, 733), CF Mendoza 18.9.50 (J 1952 I, 330)

aber CF BA 16. 10. 46 (L 44, 367)

27. 2. 1935 F 172, 220

Im Ausland geborener Sohn gebürtiger Argentinier darf nicht staatenlos werden durch Optionsverweigerung

3. 12.1937 L 8, 1059

Russe einbürgerbar, obwohl Geburtsurkunde mangels Beziehungen zur SU nicht legalisierbar.

Dito: 3. 12. 37 (L 9, 134), CF Rosario 3. 7.40 (L 19, 118),

CF Mendoza 10. 5. 49 (L 56, 7)

15. $7.1938 \quad \mathrm{~F} 181,175=\mathrm{L} 11,329$

Das arg. Recht erlaubt keine Doppelstaatigkeit: Eingebürgerter, der $\mathrm{Paß}$ seines früheren Staates benutzt, verzichtet damit stillschweigend auf seine argentinische StA.

Dito: 25. 11. 38 (F 182, $289=\mathrm{L} 12,1002$ ), CF La Plata 28. 4. 39

(L 14, 1025), O. G. 24. 5. 67 (s. u., aber Ausnahme) 
31. 3. 1939 F $183,241=\mathrm{L} 14,242$

Für Einbürgerung ist inländischer Wohnsitz nötig

dito: 1879 (F 21, 439), 1880 (F 22, 154), 1898 (F 77, 250), 1908 (F 110, 275)

4. 8. $1939 \mathrm{~F} 184,324=\mathrm{L} 15,701$

Verlust der Einbürgerung bei Wehrvergehen (Art. 22 Ley 11386) ist verfassungsmäßig

6. 8. $1940 \mathrm{~F} 186,130=\mathrm{L} 17,760$

Wehrdienst von Doppelstaatern gemäß Vertrag mit Italien von 1938 dito: 14. 5. 45 (F 201, 488), 1947 (F 206, 218)

9. 5. 1941 F $189,331=\mathrm{L} 22,832$

Option bei Auslandsgeburt auf Antrag, vgl. CF BA 1. 12. 49

(L 57, 713): Erwerb durch Geburt und Option

12. 9. 1941 F $190,517=$ L 23, 879

Im Ausland geborene Söhne eingebürgerter Argentinier haben keinen Options anspruch, nur bei gebürtigen.

24. 9. 1941 F $190,566=\mathrm{L} 24,946$

Keine Einbürgerung Unzivilisierter, insb. Analphabeten

dito: CF BA 7. 5. 41 (L 23, 207 m. Anm.), aber O. G. 21. 6.48 (s. u.)

29. 3. $1944 \mathrm{GdF} 170,181=\mathrm{L} 34,288$

Einbürgerung ist bei Betrug nicht unwiderruflich, wie CF meinte

3. 7. 1944 F $199,165=\mathrm{L} 35,198$

Kein Erwerb arg. StA durch Ehe. Option bei Auslandsgeburt.

20. 12. 1944 F 200,431 dito: 3. 7.44 (F 199, $179=\mathrm{L} \mathrm{35,199)}$

Trotz Einbürgerungsverbot im D 6605 von 1943 ist Berichtigung früherer Einbürgerungsurkunden möglich

9. 11.1945 F $203,185=\mathrm{L} 40,886$

Einbürgerungsverlust wirkt zurück bei Treubruchsakten, jedoch nicht bei 2 Jahren Auslandswohnsitz und Annahme ausländischer Ämter. Daher kein Verlust bei Tätigkeit in ausländischer Gesandtschaft, wenn genehmigt nach Erlaß von D 6605/1943

22. 2. 1946 F $204,188=\mathrm{L} 41,737$

Auf Eingebürgerten, der Leistung des Staatsbürgereids einige Jahre lang verschleppte, ist die Einbürgerungssperre für feindliche Ausländer (D 6605/1943) anwendbar.

12. 4. 1946 F $204,418=\mathrm{L} 42,387$

Einbürgerungssperre des D 6605/1943 gilt auch nach der Kapitulation Deutschlands und Japans noch weiter.

Dito sogar noch später: 13. 9. 48 (GdF 194, 601), CF BA 31. 3. 49 (L 55, 336 = Clunet 1950/51, 326)

Vor dem D 6605 war Einbürgerung eines Italieners nicht neutralitätswidrig (CF La Plata 11. 12. 42: L 36, $747 \mathrm{~m}$. Anm.)

In Deutschland geborene Russenkinder fallen nicht unter die Sperre (CF Rosario 8. 7. 49: J 1950 I, 156)

21. 6. 1948 F 211, 376

Auch Analphabeten können eingebürgert werden. Weder Verfassung noch StAG fordern, daß Bürger lesen und schreiben können.

Dito: CF Mendoza 1. 10. 42 (L 28, 799),

CF Resistentia 30. 6.59 (J 1959 V, 541)

19. 7.1948 F 211, 714 = L 51, 663

Nicht verfassungswidrig ist die Beweislast für Auslandsgeburt des Optanten 
24. 11. 1948 F 212, $321=$ L 54, 264

Einbürgerung ist bei Betrug nichtig

6. 12. 1948 F 212, $384=\mathrm{L} \mathrm{55,254}$

Einbürgerung darf nicht deshalb abgelehnt werden, weil der Betr. dadurch leichter Arbeit erlangen wollte.

15. 6. 1949 F 214,61

Inwieweit hindern frühere Straftaten Einbürgerung?

Dito: 13. 3. 50 (F 216, 160), 3. 12. 51 (F 221, 442), 14.8.52 (F 223, 314),

28. 5.53 (F 225, $714=\mathrm{J} \mathrm{83,5),} \mathrm{31.} \mathrm{5.} 53(\mathrm{~F} \mathrm{225,} 578=\mathrm{L} 71,266)$,

13. 5.54 (F 228, 640), 19. 8. 54 (F 229, 594), 15. 11.54 (F 230, 244),

3. 8. 56 (F 235, 496), 23. 9. 59 (s. u.), 2. 8. 61 (F 250, 375), 25. 9.61 (s. u.)

14. 9. 1950 F $217,888=\mathrm{L} 61,267$

Nachweis des StA-Erwerbs durch Geburt darf erleichtert werden, nachdem Verfassung Art. 31 automatischen Erwerb durch Wohnsitz vorsieht

dito: CF Rosario 31. 5. 50 (L 59, 552)

13. 11. 1950 F 218, $490=\mathrm{L} 61,542=\mathrm{GdF} 202,253=$ Clunet 79, 936

Art. 31 der Verfassung betr. automatische Ersitzung der StA ist mangels der vorgesehenen Ausführungsbestimmungen noch kein geltendes Recht. Daher kann der Betreffende als Ausländer ausgewiesen werden.

Dito: CF Bahia Blanca 24. 7. 50 (J 1950 IV, 12), CF BA 27. 12. 49 (L 58, 68), O. G. 7. 5. 51 (F 219, 315), 26. 2. 53 (F 225, 85) - aber 14. 9.50 (s. o.) und 9. 12.54 (s. u.)

21. 6. 1951 F 220,278

1918 Eingebürgerter verliert StA durch Wehrvergehen ohne Rücksicht auf Art. 31 der Verfassung, da Verlust nach Art. 36 und 22 Ley 11386 ohne Rücksicht auf Art des Erwerbes erfolgt.

27. 6. 1951 F 220, 516

Inwieweit Einbürgerung Fähigkeit, sich selbst zu unterhalten, voraussetzt.

Dito: CF Rosario 29. 10. 41 (L 24, 417), 2. Urteil vom 27. 6. 51 (F. 220, 518), 27. 12. 54 (F 230, 597)

22. 11. 1951 F 221, 245=L 65, 145

Option des im Ausland Geborenen erst ab 18 Jahre

29. 11. 1951 F 221, 335 = L 66, 90

Inwieweit der Einzubürgernde natürliches Glied der Gemeinschaft werden muß, insbesondere im Alter.

Dito: Urteile vom gleichen Tage (F 221, $399=\mathrm{L} 66,95)$

(F 221, $338=\mathrm{L} 65,330)$

3. 12. 1951 F 221, $413=\mathrm{L} 66,86$

Notwendigkeit zur Option bei Auslandsgeburt ist als Ausnahme vom automatischen Erwerb zulässig.

21. (22.) 2. 1952 F 222, $85=\mathrm{L} 65,769=\mathrm{J} 1952 \mathrm{III}, 330 \mathrm{~m}$.

Anm. Grossmann

$\mathrm{Daß}$ Einzubürgernder gute Führung besitzen muß, steht zwar nicht im Gesetz, ergibt sich aber von selber. StA entziehen, wenn nicht vom Richter des Wohnsitzes erlangt.

Dito zu Führung: CF Rosario 27. 5. 41 (L 23, 311), O. G. 10. 8. 56 (F 235, 622), 20. 3. 59 (F 243, $202=\mathrm{L} \mathrm{96,} 448$ m. Anm.), 5. 8. 60 (F $247,444=\mathrm{L} 102,520 \mathrm{~m}$. Anm. Newa) 8. 2. 61 (F 249, 24 $=\mathrm{L} 105,554 \mathrm{~m}$. Anm. Newa) 
26. 2. 1953 F $225,85=\mathrm{J} 83,1$

Art. 31 der Verfassung ist noch nicht wirksam. Art. 22 Ley 11386 gilt weiter und führt zum StA-Verlust.

18. 3. 1954 F 228, $140=\mathrm{L} 74,713$

Keine StA-Option für Adoptierte, deren einer Adoptivelternteil Argentinier ist. Wahlgesetz stellt Adoptierte zwar gleich, aber kann nicht StA gewähren.

9. 12.1954 F 230,480

Einbürgerung wegen kleiner Mängel zu widerrufen, ist unrecht, wenn Fünfjahreswohnsitz für automatischen Erwerb nach Art. 31 erfüllt wäre.

16. 6. 1958 F 241, $31=\mathrm{L} 94,455 \mathrm{~m}$. Anm. Newa

Wer 1945 strafverurteilt, aber seitdem straffrei, kann eingebürgert werden, Art. 10 (d) der DVO von 1931 ist unanwendbar.

23. 9. 1961 F 244, $496=\mathrm{L} 99,728 \mathrm{~m}$. Anm. Newa

Die DVO von 1931 gilt noch heute unverändert.

25. 9. 1961 F $250,758=\mathrm{L} 106,95$

Ausschluß der Einbürgerung bei Delikten ist nicht verfassungswidrig (Art. 86, 2)

8. 11.1963 F $257,105=\mathrm{L} 113,326$

Wiedererwerb Amnestierter aus Wehrvergehen ist nicht auf Kongreß beschränkt (Art. 9 StAG)

24. 5. 1967 L $127,246=$ Clunet 1968, S. 742

Gebrauchmachen von ursprünglicher (italienischer) StA (Paß) durch Eingebürgerten führt zum Verlust der argentinischen StA.

Dies Urteil hebt auf das gegenteilige der Vorinstanz, nämlich der CF La Plata vom 19. 5. 65 (L 118, $692=$ Clunet 1967, S. 960).

Wenn im 2. Weltkrieg britischer $\mathrm{Paß}$ benutzt wegen Ausnahmesituation, dann kein StA-Verlust (CF BA vom 18. 4. 49: L 55, 621).

Literatur

Arbo, $\mathbf{H}$.

Bosch, B.

Cabaleiro, E.

Castro Dassen, H. N.

Dana Montaño, S. M.

Diaz Cisneros, C.

Ecudero, M.

González Calderón, J. A.

Grossmann, C.

Gschwind, F. J.

Justo, A. M.

Machado Doncel, J.

Maxl, S.

Medrano, S. W.

Rauchaupt, F. W. v.
La naturalización de los extranjeros

in: JA 1925, Bd. 18, Doctrina S. 63-68

Concessión de cartas de ciudadaniá por los provincias

in: Rev. del Col. de Abogados de Buenos Aires 1945, Nr. 3, S. 521

Doble nacionalidad

in: Revista general de législación y jurisprudencia 1962, S. 7

Ley de ciudadaniá y facultades extraordinarias

in: La Ley 1956, Bd. 82, S. 816-818

El régimen argentino sobre nacionalidad y ciudadaniá

in: La Ley 1956, Bd. 84, S. $925-941$

Différenciatión juridique et politique de la citoyenneté et de la nationalité in: Cahiers de législation et de bibliographie juridique de l'Amérique latine (Paris), Heft 3 (1949), S. 13-24

La nacionalidad de las personas en los tratados de la República Argentina

con España in: Annuario hispano-luso-americano de derecho int. (Zaragoza) 1963, Bd. 2, S. $187-194$

Prueba de la extranjeriá a los efectis de obtener la nacionalidad argentina. Naturalización automatica.

in: JA 1951 III, Doctrina S. 57-61

Curso del derecho constitucional

2. Auflage, Buenos Aires 1958 (StA: S. 177-180)

Cancelación de la carta de ciudadaniá

in: JA 1952 III, S. 330-338 (zum Urteil O. G. vom 21. oder 22. 2. 1952)

La ciudadaniá y naturalización segun el derecho argentino, Santa Fé 1941

Apuntaciones sobre la ciudadaniá y naturalización

in: La Ley 1954, Bd. 75, S. 893-896

Ciudadaniá y naturalización

in: La Ley 1937 , Bd. 7 , Doctrina S. $83-85$

Algunas preceptos que debe contener la ley reglementaria de la ciudadaniá

in: Gaceta del Foro, Bd. 199, S. 385

in: Gaceta del Foro, Bd. 199, S. 385 sociales en el debat sobre ciudadaniá en 1826

in: Rev. de la Fac. de Der. y Sc. Soc. (BA) 1953, S. 1155

Nationalität und Staatsbürgerschaft. Die argentinische Doctrina de Garay

in: Europäische Gespräche 1926, S. 5-14 
Romero del Prado, V. N. La naturalización de los extranjeros en la Constitución de 1949

in: La Ley 1950, Bd. 59, S. 991-995

La Constitución Nacional de 1949 y los principios fundamentales en materia de nacionalidad

in: La Ley 1950 , Bd. 60 , S. $790-793$

El derecho de opción en materia de nacionalidad. Su incorporación en la Constitución Nacional de 1949

in: La Ley 1950 , Bd. 60 , S. 945-948

La reforma de la Constitución Nacional de 1853. La Constitutión de 1949. Como la sido incorporado en estra ultima el derecho de opción en materia de nacionalidad

in: La Ley 1951, Bd. 61, S. 849-854

Iniciativas y proyectos parlamentarios en favor de la naturalización automatica y de la opción tácita

in: La Ley 1951, Bd. 62, S. 919-924

Naturalización automatica.

in: La Ley 1951, Bd. 62, S. 972-980

La doble nacionalidad: La nacionalidad ibero-americana, la nacionalidad hispano-argentina, la nacionalidad continental-americana

Ruiz Moncada, F.

in: La Ley 1962, Bd. 108, S. 1114-1121

Hacia un estatuto que establezca la condición jurídica de los nacionales en la comunidad Hispano-Luso-Americano

in: La Ley 1956, Bd. 83, S. 959-961 (Bericht über einen Kongreß)

Sallustro, O.

Conflictos de ciudadaniá y doble ciudadaniá.

Buenos Aires 1960

La prueba de la calidad de extranjero en el juicio por carta de ciudadaniá. Prueba supletoria del nacimiento

in: La Ley 1949, Bd. 55, S. 134 (zu CF Rosario vom 26. 2. 1949)

Ciudadaniá y naturalización. Conducta irreprochable del petionante. La opción y la nueva Constitucion Nacional. Adquisitión automatica de la nacionalidad

in: Boletino del Instituto de Enseñanza práctica (Buenos Aires) 1951, S. $447 \mathrm{ff}$.

\section{BARBADOS}

Úbersicht über Gesetze (1892-1967)

in: StAZ 1968, S. 25

\section{BOLIVIEN}

Ubersicht über Rechtsquellen (Verfassungen, Gesetze und Verträge) von 1826-1961 in: SGS Bd. 24, S. 47-51; Lit. S. 89-90

\section{Gesetze:}

Verfassung vom 2. 2. 1967

StAB: Art. $36-39$

Ab 6. 11. 1964 war wieder die Verfassung von 1947 an Stelle der von 1961 in Kraft gewesen

Amtlicher Text: G. O. vom 17. 4. 1967

Holländische Übersetzung: Adriaansen, Bb 1

\section{Verträge:}

Doppelstaater-Vertrag mit Spanien vom 12. 10. 1961

Inkrafttreten: 31.3 .64

Amtlicher Text: BOE vom 14. 4. 64

Spanischer Text: EJE 1964, S. 1061

\section{Literatur}

A statement of the laws of Bolivia in matters affecting business, Washington 1962, StA: S. 13-24

\section{BRASILIEN}

Übersicht über Rechtsquellen (1824-1949)

in: SGS Bd. 19, S. 85-89; Lit. S. 125-126

\section{Gesetze:}

17. 3. 1944 Portaria Nr. 7620 betr. DVO zum StAG vom 25. 4. 1938

Amtlicher Text: D. O. vom 22. 3. $44=$ vom 27. 3.44 (Ber.: 4. 4. 44)

Portugiesischer Text: RDT Bd. 149, S. 836 
4. 7. 1957 Gesetz Nr. 3192 betr. Änderung des StAG von 1949

ändert Art. 7, 8 VII $₫ 1$, 9 VI, 10, 15, 16, 19, 34, 35, 43

Amtlicher Text: D. O. Nr. 153 vom 6. 7. 1957

Englische Übersetzung: YHR 1958, S. 15 (Auszug)

Portugiesischer Text: RDT Bd. 264, S. 883

Coleção das leis 1957 V, S. 23

Spanischer Text: BLE 1959 (Bd. 26), S. 53-70

(Neuabdruck des StAG i. F. von 1957)

Französische Übersetzung: TLE 1958, Nr. 3, S. 3

(Neuabdruck des StAG i. F. von 1957)

18. 12. 1959 Gesetz Nr. 3696 betr. Einbürgerung der Frau, die im Ausland einen brasilianischen Diplomaten heiratet

Amtlicher Text: D. O. vom 22. 12. 59, S. 26633

Portugiesischer Text: Coleção das leis 1959 VII, S. 52

RDT Bd. 293, S. 763

14. 9. 1964 Gesetz Nr. 4404 betr. die StA minderjähriger im Inland wohnender

Kinder eingebürgerter Brasilianer

aufgehoben: 20. 10. 66 (s. u.)

Amtlicher Text: D. O. vom 18. 9.64

Holländische Übersetzung: Adriaansen, Brasilien

Portugiesischer Text: Coleção das leis 1964 V, S. 107

20. 10. 1966 Gesetz Nr. 5145 betr. Änderung des StAG von 1949

ändert Art. 3, 4, 8. Hebt auf Gesetz vom 14. 9.64 (s. o.)

Amtlicher Text: D. O. vom 24. 10. 66 (Ber.: 27. 10.)

Portugiesischer Text: Coleção das leis 1966 VII, S. 273

RDT Bd. 375, S. 394

12. 12. 1966 Bundesverfassung

(24. 1. 1967) Inkrafttreten: 15. 3. 1967

StAB: Art. 140-141

Englische Ubersetzung: CP Inf 1968, S. 2 und 54

Französische Übersetzung: Inf CP 1968, S. 2 und 54

NE Nr. 3512 vom 31. 8. 68

17. 10. 1969 Bundesverfassung

Inkrafttreten: 30. 10. 1969

StAB: Art. $145-146$

Portugiesischer Text: Journal do Brasil v. 18. 10. 69, S. 13

\section{Verträge:}

6. 8.1876 Konsularvertrag mit Italien

StAB: Art. 19

Amtlicher Text: G. U. vom 31. 8. 1877

Italienischer Text: Martens, N. R. G., 2. Serie, Bd. 4, S. 261

27. 4. 1908 Einbürgerungsvertrag mit den USA

gekündigt von Brasilien ab 14. 12. 1951 (State Papers Bd. 158, S. 730)

Amtlicher Text: D. O. von 1909 (Dekret Nr. 2116 vom 8. 10. 09)

Englischer Text: AJIL 1910, Suppl. S. 262

2. 3. 1929 Modus vivendi über Doppelstaater mit Italien

Englischer Text: Flournoy, 1929, S. 709

6. 9. 1958 Übereinkommen mit Italien über Wehrdienst der Doppelstaater

Inkrafttreten: 15. 1. 1965

Amtlicher Text: D. O. Nr. 163 vom 9. 6. 1965

(Dekret Nr. 56417 vom 4. 6. 65)

Italienischer Text: D. I. 1967 II, S. 177 


\section{Entscheidungen}

Im folgenden sind aus der Revista dos Tribunais die StA-Entscheidungen seit dem

2. Weltkrieg verzeichnet.

Die Abkürzungen bedeuten:

LS: nur Leitsatz abgedruckt, nicht das Urteil

P: $\quad$ Tribunale São Paulo

R: Tribunale Federale de Recursos

S: $\quad$ Supremo Tribunale Federale

25. 10. 1943 152, 772 S Einbürgerung minderjähriger Italiener nach Verfassung von 1891

8. 12. 1944 155, 283 P Automat. Erwerb nach Art. 69 IV Verf. v. 1891 Auslegung von Art. 25 des StAG vom 25. 4.38

22. 1. 1945 162, $352 \mathrm{~S}$ Automat. Erwerb nach Art. $69 \mathrm{~V}$ Verf. von 1891 durch Portugiesen

21. 9. 1945 160, $776 \mathrm{P}$ Automat. Erwerb durch Portugiesen; Art. 25 $\operatorname{des}$ St AG vom 25. 4.38

19. 8. 1947 170, 212 P Option bei Auslandsgeburt (Art. 129 II Verf. von 1946)

21. 10. $1947171,625 \mathrm{P}$ Option bei Auslandsgeburt (Art. 69 II Verf. von 1891)

20. 12. 1947 177, 208 P Automat. Erwerb nach Art. 69 IV Verf. von 1891 durch Italiener

26. 12. 1947 192, 879 S Automat. Erwerb nach Art. 69 V Verf. v. 1891 durch Portugiesen

10. 12. 1948 179, 773 P Option bei Auslandsgeburt (Art. 69 II Verf. v. 1891)

3. 10. 1949 188, 467 R LS: Verlust deutscher StA einer Frau und Staatenlosigkeit antragsgemäß anerkannt

27. 3. $1950186,648 \mathrm{P}$ Unterschiede "ciudadania " und "nacionalidade"

6. 9. 1950 195, 509 R Automat. Erwerb nach Art. 69 Verf. v. 1891 durch Polen

26. 9. 1950 201, 565 R LS: Automat. Erwerb nach Art. 69 Verf. v. 1891

21. 8. 1951 208, $642 \mathrm{R}$ LS: dito

1. 6. 1951 203, 617 R LS: dito

17. 9. 1951 203, $617 \mathrm{R}$ LS: Staatenlosigkeit

16. 11. 1951 226, 581 S LS: Automat. Erwerb

21. 12. 1951203,617 R LS: Gebrauchmachen von ursprünglicher StA vor Verfassung von 1934

21. 10. 1953233,538 R LS: Zwangseinbürgerung ist unzulässig

14. 1.1956 249, 460 Trib. Just. Parana Option eines Italieners gemäß Art. 1 II des StAG v. 1949

18. 1. 1957 262, $334 \mathrm{P}$ Option bei Auslandsgeburt

18. 4. 1958 277, 337 P Portugiese, der StA nach Art. 69 Verf. v. 1891 erwarb, behält sie, wenn er nie Verzicht behauptete

27. 10. 1958 298, 761 S LS: Option, Zuständigkeit

12. 1. 1959 294, 727 S LS: Widerruf der Einbürgerung

11. 7. $1961334,536 \mathrm{~S}=350,586 \mathrm{~S}$

Option bei Auslandsgeburt nicht ex tunc

29. 3. $1963356,133 \mathrm{P}$ in Brasilien Geborener, der sich beim deutschen Konsul registrieren ließ, kann nicht mehr für brasilianische StA optieren

Literatur (neue)

Alexandre, F.

Alves Barbosa, A. A.

Carvalho, A. D. de

Monteiro, W. de Barros

Naturalizados e outros aspectos da nacionalidade

Rio de Janeiro 1956, 242 S.

Opção de nacionalidade

in: RDT, Bd. 310, S. 80-81 (Gutachten von Juli 1961)

Naturalização

in: Repertorio Enciclopédico do Direito Brasileiro, Bd. 34 (1966), S. 32-85

Da nacionalidade e da Cidadania em face da nova Constituição

in: Revista de direito administrativo 1968, S. 13-24 
Oliveiro, A. Goncalves de

Penna Marinho, I

Sachse

Tenorio, $\mathrm{O}$.

Valladão, $\mathbf{H}$.

o. V.
Perda da nacionalidade brasileira pela pretação de serviço militar no extrangeiro

in: Revista Forense (Rio) 1958, Bd. 182, S. 13-15

Nacionalidade brasileira. Perda por quem prestou servico militar em pais extrangeiro

in: RDT 1956, Bd. 254, S. 88-89 (Gutachten vom 26. 10. 1956)

Nacionalidade brasileira. Perda por naturalizado que permaneceu por meis de 6 meses no estado de israel - inadmissilidade

in: RDT 1957, Bd. 258, S. 91-94 (Gutachten vom 17. 1. 1957)

Adoção de nacionalidade no direito brasileiro

in: Revista brasileira de politica int. 1961, Nr. 16, S. 36-53 (zum Senatsentwurf eines neuen StAG)

Tratado sobre a nacionalidade, Bd. 3, Rio de Janeiro 1957 (Brasil. StAR)

Brasilianisches StA-Recht

in: StAZ 1969 , S. 80

A opcão da nacionalidade brasileira

in: Arquivos 1966, Nr. 97

Direitos políticos dos brasileiros naturalizados

in: RDT 1958, Bd. 277, S. 7-11

Proteção diplomatica como amparo dos direitos de homen - Naturalização e irretroatividade no direito brasileiro

in: RDT 1954, Bd. 221, S. 25-39

Nacionalidade, no direito brasileiro

in: Arquivos $1959, \mathrm{Nr} .70$

Naturalizaçao - interpretação do Art. 8 item VI da Lei Nr. 818 de 1949 Penan "in abstracto" e penan "in concreto"

in: Arquivos 1962, Nr. 81, S. 55

Dilermando de Castello Cruz, Opção inconstitutional pela nacionalidade brasileira - criação fraudulenda da brasileiros natos

in: Arquivos 1963, Nr. $86=$ Rev. Trib. 1963, S. 336

Opção de nacionalidade

in: RDT 1961, Bd. 313 , S. $669-670$

Nachträge zu älterer Literatur:

Arbo, $\mathrm{H}$.

Flores, L. L.

Rodrigo Octavio

Silveira, A. X. de

o. V.

o. V.
Ley del Brasil sobre ciudadania y naturalizacion

in: Jurispr. Argentina 1925, Bd. 18, S. 64 Doctr.

A nacionalidade dos filhos de pae portuguez nazidos no Brasil,

Rio Grande do Sul 1895

La double nationalité et la parte de la nationalité specialement au point de vue de la législation du Brésil

in: Revue de l'inst. de droit comp. 1913, S. 299-318

in: Revue de

Naturalização
Rio de Janeiro 1920

Nacionalização dos portugueses no Brasil

Rio de Janeiro 1890

Martens, N. R. G., 2. Serie, Bd. 19, S. 672-692

\section{CANADA / KANADA}

A. Acts

Nicht aufgeführt sind die Private Acts Oberkanadas und Kanadas betreffend Einzeleinbürgerungen.

1828 Gesetz von Oberkanada betr. Einbürgerung

(9 Geo. 4, c. 21)

26. 5. 1826 Britisches Gesetz betr. Gültigkeit oberkanadischer Einbürgerungen u.a. (7 Geo. 4, c. 68)

1830 Zwei Gesetze von Oberkanada betr. Verlängerung der Frist zur

u. 1838 Eidesleistung der Eingebürgerten

(1 Wm. 4, c. 7 ; 2 Vict., c. 20)

1830

Gesetz von Unterkanada betr. Einbürgerung

(1 Wm. 4, c. 51)

27. 8. 1841 Kanadisches Gesetz betr. Einbürgerung

(4/5 Vict., c. 7)

Die beiden Provinzen Ober- und Unterkanada waren $1840 \mathrm{zu} \mathrm{Ka-}$ nada vereinigt worden.

Englischer Text: State Papers, Bd. 68, S. 1322

1846

Gesetz betr. Verkürzung der Wohnsitzfrist

bei Einbürgerung von 7 auf 5 Jahre

(9 Vict., c. 107) 
30. 5. 1849 Gesetz betr. Einbürgerung

(12 Vict., c. 197)

Englischer Text: State Papers, Bd. 72, S. 652

1855 Gesetze betr. Verkürzung der Wohnsitzfrist

u. 1859 (18 Vict., c. 6 und 22 Vict., c. 1)

1859 Konsolidiertes Einbürgerungsgesetz

(22 Vict., c. $8=$ Cons. Stat. Can., c. 8)

Englischer Text: Foreign Relations 1873 II, S. 1256 (Auszug)

$1868 \quad$ Aliens and Naturalization Act

(31 Vict., c. 66)

Englischer Text: State Papers, Bd. 65, S. 1201

14. 4.1871 Gesetz betr. frühere Einbürgerungen

(34 Vict., c. 22)

Englischer Text: State Papers, Bd. 65, S. 1331)

23. 5. 1873 Gesetz betr. Erstreckung der Einbürgerungsgesetze des Dominions Kanada auf Britisch Columbia und Manitoba

(36 Vict., c. 36)

Englischer Text: State Papers, Bd. 65, S. 1333

21. 3. 1881 Dominion Naturalization Act

(44 Vict., c. 13)

65 Sections mit Schedules A-I

aufgehoben: 1. 1.1915

Amtlicher Text: Rev. Stat. 1887, c. 113; 1906, c. 77

Englischer Text: State Papers, Bd. 72, S. 643 (Fass. 1881)

$1883 \quad$ Gesetz betr. Geltung des Einbürgerungsgesetzes

(46 Vict., c. 31 )

15. 5. 1902 Naturalization Amendment Act

(2 Edw. 7, c. 23: Allgemeine Ergänzungen des Gesetzes von 1881)

Englischer Text: State Papers, Bd. 95, S. 307

13. 8. 1903 Naturalization Amendment Act

(3 Edw. 7, c. 38: ändert Sect. 11, 12, 18, fügt ein 11A, ändert Schedule

Form F, ändert Sect. 5 von 1902)

Englischer Text: State Papers, Bd. 97, S. 609

10. 8.1904 Naturalization Amendment Act

(4 Edw. 7, c. 25: ändert Sect. 16)

20. 7.1905 Naturalization Amendment Act

(4/5 Edw. 7, c. 25 : ändert Sect. 11A und Form A)

Englischer Text: State Papers, Bd. 98, S. 576

30. 1. 1907 Naturalization Amendment Act

(6/7 Edw. 7, c. 31 : allgemeine Ergänzungen, zusätzliche Form 1-4)

Englischer Text: State Papers, Bd. 100, S. 654

1907 Statute Law Amendment Act

(6/7 Edw. 7, c. 45: betr. Ausdehnung des Einbürgerungsgesetzes auf Saskatchewan und Alberta)

20. 7.1908 Naturalization Amendment Act

(7/8 Edw. 7, c. 48 : ändert Sect. 16, 47, 54)

Englischer Text: State Papers, Bd. 101, S. 782 
Immigration Act

In diesem Gesetz Kanadas erscheint zum ersten Mal der Ausdruck "Canadian Citizenship".

Immigration Act: R. S. 1927, c. 93

12. 6. 1914 Naturalization Act

Inkrafttreten: 1.1.1915

(4/5 Geo. 5 , c. 44$)$

Dies ist eine Übernahme des Britischen StA-Gesetzes von 1914.

Mit 36 Sections und 3 Schedules.

Amtlicher Text: Rev. Stat. 1927, c. 138, S. 2831

Englischer Text: State Papers, Bd. 107, S. 416

Flournoy, S. 75

Parry, S. 452 (ohne Sect. 18-34 und Schedules)

22. 8. 1914 Naturalization Amendment Act

ändert Sect. 2, 10, 25

(5 Geo. 5, Sess. 2, c. 7)

Englischer Text: State Papers, Bd. 108, S. 201

1919 Naturalization Amendment Act

(9/10 Geo. 5 , c. 38$)$

1920 Naturalization Amendment Act

$(10 / 11$ Geo. 5 , c. 59$)$

3. 5. 1921 Candadian Nationals Act

(11/2 Geo. 5, c. 4)

Amtlicher Text: Rev. Stat. 1927, c. 21, S. 347

Englischer Text: State Papers, Bd. 116, S. 284

Flournoy, S. 86

Französische Übers.: Bourbousson, S. 299

1923 Naturalization Amendment Act

(13/4 Geo. 5, c. 60: ändert Sect. 2-3)

1931 Naturalization Amendment Act

Inkrafttreten: 15.1.1932

(21/2 Geo. 5, c. 39: ändert Sect. 13)

Französische Übersetzung: Bourbousson, Anhang S. 12

27. 6.1946 Canadian Citizenship Act, Nr. 15 (Sess. 1)

Erste Bill vom 22. 10. 1945 (Off. Rec. of Debates H. C. 1945, S. 1335).

(10 Geo. 6, c. 15 [Sess. 1])

Amtlicher Text: Rev. Stat. 1952, c. 33, S. 1607

Deutsche Übersetzung: Bergmann, Kanada, S. 1 (ohne Sect. 26-38, 41-43)

Englischer Text: LCN, S. 69 (Fass. 1951)

LCN, Nachtrag, S. 15 (Fass. 1954)

Adriaansen, c. 1

Parry, S. 472

Canadian Citizenship Act, Ottawa 1969 (Fass. 1967)

Französische Übersetzung: Bettoni, Bd. 1, S. 428

TLE 1951, Nr. 3, S. 11

Spanische Übersetzung: Bol. de Leg. Extranj. 1951

(Bd. 17), S. 227, 375, 467

Holländische Übersetzung: Adriaansen, c. 21

25. 3. 1949 Statute Law Revision (Newfoundland) Act

StAB: Sect. 46

1950

(13 Geo. 6, c. 6)

Act

(14 Geo. 6, c. 15) 
30. 6. 1950 Canadian Citizenship Amendment Act

(14 Geo. 6, c. 29 (Sess. 1)

31. 5. 1951 Canadian Citizenship Amendment Act

(15 Geo. 6, c. 12: ändert Sect. 19)

Französischer Text: TLE 1951, Nr. 3, S. 28

14. 5. 1953 Canadian Citizenship Amendment Act

(1/2 Eliz. 2, c. 23: ändert Sect. 2, 4-6, 9-11, 18, 34, 38, in je zwei Fassungen entsprechend dem Inkrafttreten der R. S.)

Amtlicher Text: Statutes 1952/3, S. 89

10. 6. 1954 Canadian Citizenship Amendment Act

(2/3 Eliz. 2, c. 34: ändert Sect. 10 und 44)

Amtlicher Text: Statutes 1953/4, S. 175

7. 6. 1956 Canadian Citizenship Amendment Act

(4/5 Eliz. 2, c. 6: ändert Sect. 2, 9, 10, 26, 28, 30-32, 34)

Amtlicher Text: Statutes 1956, S. 33

Englischer Text: LCN, Nachtrag, S. 30

6. 9. 1958 Canadian Citizenship Amendment Act

(7 Eliz. 2, c. 24: ändert Sect. 10, 19, fügt ein 19A)

Amtlicher Text: Statutes 1958, S. 155

1966

Canadian Citizenship Amendment Act

$1966 / 7$, c. 25

ändert Sec. 2 (1)

Amtlicher Text: Statutes 1966/7, S. 41

1967

Canadian Citizenship Amendment Act

$1967 / 8$, c. 4

Inkrafttreten: 7. 7.1967

ändert: Sec. 10 (1, b-e), 10 (8, a), 12, 19 (1-2), 20 (2), 30, 31, 34 (1), 39 (1 u. 6), 41 ;

hebt auf Sec. 18;

fügt ein Sec. 30A, 39A, 39B, 39C, 41 A, 42A

B. Verordnungen

17. 6. 1931 P.C. 1378 betr. Einbürgerung von Japanern, nur bei Verlust ihrer StA

13. 8. 1934 P. C. 1760 betr. Einbürgerung von Chinesen und Japanern

ändert die Order von $1931 \mathrm{ab}$

Englischer Text: Univ. Toronto LJ 1935/6, S. 137

19. 11.1946 P. C. 4761 betr. StA

11. 2. 1947 P. C. 502 betr. StA

8. 7.1947 P. C. 2666 betr. StA

8. 7. 1948 P.C. 2886: Canadian Citizenship Regulations

Amtlicher Text: OIC, P. C. 2886 von 1948

26. 5. 1953 P.C. 862: Canadian Citizenship Regulations

Inkrafttreten: 1.6. 1953

Amtlicher Text: OIC, P. C. 862 von 1953

Englischer Text: Adriaansen, c. 13

18. 8. 1954 P. C. 1190: Canadian Citizenship Regulations

Amtlicher Text: Gazette Nr. 17 vom 18. 9. 1954, S. 1077 (SOR 364)

Englischer Text: Statutory Orders und Regulations Comolidation 1955, Bd. 1, S. 589

1964 Canadian Citizenship Regulations Amendment

Amtlicher Text: Gazette Nr. 11 vom 10. 6. 1964 (SOR 199) 
28. 8. 1968 P.C. 1703: Canadian Citizenship Regulations

Inkrafttreten: 1. 10. 1968

Englischer Text: Canadian Citizensphip Act, Ottawa 1969, S. 26

\section{Gliedstaaten}

Von den übrigen „Provinzen“ gibt es keine StA-Bestimmungen. Ober- und Unterkanada siehe unter Teil A. Nicht aufgeführt sind hier die Private Acts, durch die Einzelbürgerungen erfolgten in Neuschottland und Prinz-Eduard-Insel.

I. Britisch Columbia

14. 5. 1859 Proklamation betr. Einbürgerung

Amtlicher Text: Rev. Stat. 1871, App.

Englischer Text: Publ. Pap. on British Columbia 1860, Teil 3

$1867 \quad$ Naturalization Ordinance, Nr. 37

23. 5. 1873 Kanadisches Gesetz betr. Erstreckung der kanadischen Einbürgerungsgesetze auf Britisch Columbia und Manitoba

Damit wird die Ordinance von 1867 noch ein Jahr verlängert.

(36 Vict., c. 36)

Englischer Text: State Papers, Bd. 65, S. 1333

II. Neubraunschweig

1841 Einbürgerungsgesetz

(4 Vict., c. 45)

1845 Gesetz über die Verlängerung der Frist

des Einbürgerungsgesetzes

$1846 \quad$ Gesetz über die Aufhebung des Verlängerungsgesetzes

(9 Vict., c. 6)

$1850 \quad$ Einbürgerungsgesetz

(13 Vict., c. 41)

Amtlicher Text: Rev. Stat. 1854, Titel 12

1861 Gesetz zur Verkürzung der Wohnsitzfrist zur Einbürgerung

(24 Vict., c. 54)

1865 Gesetz betr. lokale Wirkung der Einbürgerung

(28 Vict., c. 5)

III. Neufundland

12. 5. 1856 Erstes Einbürgerungsgesetz

(19/20 Vict., c. 20$)$

Amtlicher Text: Consol. Stat. 1872, c. 72

Consol. Stat. 1892, c. 145

30. 5. 1891 Gesetz betr. Einbürgerung der Ausländer

Erwähnt bei Zeballos Bd. I, S. 574/ und Cahn, 3. Aufl.

1900 Gesetze über Änderung des Einbürgerungsgesetzes

u. 1908 (63 Vict., Sess. 2, c. 7; 8 Edw. 7, c. 3)

5. 6. 1915 Einbürgerungsgesetz: Gesetz betr. Übernahme

des Einbürgerungsgesetzes des UK von 1914

(6 Geo. 5, c. 11)

Amtlicher Text: Consol. Statutes 1916, c. 78

Englischer Text: Flournoy, S. 137

State Papers, Bd. 109, S. 604

4. 5. 1916 Gesetz betr. Änderung des Einbürgerungsgesetzes

Englischer Text: State Papers, Bd. 110, S. 386 
25. 3. 1949 (Kanad.) Statute Law (Newfoundland) Act

(13 Geo. 6, c. 6)

(siehe oben Kanada)

\title{
IV. Neuschottland
}

\author{
$1775 \quad$ Gesetz betr. Treueid zur Eingesessenheit \\ 1847 Gesetz, daß jeder Eingebürgerte den Eid zu leisten hat \\ (11 Vict., c. 27) \\ 1851 \\ Konsolidiertes Einbürgerungsgesetz \\ 1858 \\ (Rev. Stat. 1851, Titel 8) \\ Einbürgerungsgesetz \\ (26 Vict., c. 36) \\ Amtlicher Text: Rev. Stat., 3. Serie, Titel 9

\section{Prinz-Eduard-Insel} \\ 17. 4. 1862 Einbürgerungsgesetz \\ (25 Vict., c. 24) \\ Amtlicher Text: Laws of PEI, Bd. 2, S. 567
}

\section{Literatur}

Adriaansen

Angus, H. F.

Baty, T.

Boshowers, F.

Bourbousson, E.

Brown, M.

Clarke, A. H.

Hellendal, F.

Howell, A.

Kennedy, W. P. M.

Kennedy, W. P. M.

Mulvey, Th.

Parry, C.

Perrault, C.

Riddell, W. R.

Tory, H. M. und Hyndman, J. D.

Vallat, F. A.

Wang, E. B.

Wilkinson, W. E.

Zeballos, E.

o. V.
Nationaliteitswetgiving, Arnheim o. J., Canada, C. 33-41

The legal status in British Columbia of residents of oriental race and their descendants

in: Can. Bar Rev. 1931, S. 1-12

History of Canadian nationality

in: JC Legislation (London) 1936, (3. Serie, Bd. 18), S. 195-203

Nationalité

Brügge 1951, S. 166-171

La nationalité

Paris 1931, S. 51-54

Expatriation of infants; being a study in the conflict of laws between Canada and the U. S.

in: University of Toronto LJ 1939/40 (Bd. 3), S. 97-113

Citizenship and naturalization

in: Can. LT 1915 (Bd. 35), S. 317

Conflict of law, domicile, naturalization

in: Can. Bar Rev. 1938 (Bd. 16), S. 312-314; 1938 (50), S. 92-93

$=$ Juridical Review (Edinburgh)' (engl. Fall Gulbenkian)

Naturalization and Nationality in Canada

Toronto/Edinburgh 1884, $132 \mathrm{~S}$.

The law of nationality in Canada

Ottawa 1930

Nationality

in: University of Toronto LJ 1935/6 (Bd. 1), S. 139-141; S. 141-146:

Bill 24: Neues "Canadian Nationals Act, 1951"

Imperial Naturalization

Ottawa 1912

Nationality and Citizenship laws

London 1957, S. 431-522, 1123-1124

La nationalité [Canada]

Arthabasca 1939

The first naturalized Canadian

in: Can. Bar Rev. 1931 (Bd. 9), S. 119-121

Canadian Citizenship (Tory: Vortrag, S. 1)

Who is a citizen? (Hyndman: Memorandum, S. 10)

Ottawa 1945, 16 S. (Canadian Council of Education for Citizenship)

Nationality of married women

in: Can. Bar Rev. 1934 (Bd. 12), S. 283-292

Nationality of claims and diplomatic intervention - Canadian practise

in: Can. Bar Rev. 1965 (Bd. 43), S. 136-150

Marriage and nationality

in: Can. LT 1915 (Bd. 35), S. 906

La nationalité, Paris 1919 , Bd. 4, S. $318-329$

Allegiance and expatriation

in: Can. Bar Rev. 1927 (Bd. 5), S. 366-368

The Canadian Citizenship Act

in: Can. Bar Rev. 1947 (Bd. 25), S. 364-372

= Fortnightly LJ (Toronto) 1947 (Bd. 16), S. 221

De la situation des Français au Canada au point de vue des conflicts de nationalité

in: RDIP 1913, S. 659-663 


\section{Verträge}

Canada ist an die früheren StA-Verträge Großbritanniens gebunden. Der Haager Konvention vom 12. 4. 1930 trat Canada selbständig bei, nicht dagegen den Protokollen dazu.

Das Frauen-Abkommen vom 29. 1.1957 gilt für Canada ab 19. 1. 1960. Ein "Abkommen" mit Japan von 1934 ist kein Vertrag, sondern ein Notenwechsel. der zur Änderung der canadischen Regulations führte (s. o.).

3. 6. 1918 Vertrag Großbritanniens (Canadas) mit den USA

betr. Wehrdienst von Amerikanern und Canadiern

im anderen Land

Inkrafttreten: 30. 7. 1918

Amtlicher Text: Statutes at large, Bd. 40, S. 1624

TS (UK), Nr. 634 von 1918

1952

Englischer Text: State Papers, Bd. 111, S. 572

Vereinbarung mit Deutschland über Austausch

von Einbürgerungsmitteilungen

(Mitteilung durch Rschr. BMI vom 29. 8. 1952:

GMBl 1952, S. $240=$ StAZ 1952, S. 249)

\section{CHILE}

Übersicht über Rechtsquellen (1822-1953)

in: SGS Bd. 19, S. 160-162; Lit. S. 201-203

\section{Gesetze:}

31. 10. 1884 Gesetz betr. Angliederung der von Peru abgetretenen Provinz Tarapaca

StAB: Art. 14

Spanischer Text: H.-A. Schmidt, StA-Wechsel

bei Staatensukressionen, München 1966, S. 135

12. 9. 1957 Gesetz Nr. 12548 betr. Änderung der StAB der Verfassung

(Art. 5-6)

Amtlicher Text: D. O. vom 30. 9. 1957

\section{Verträge:}

9. 7.1928 Accord mit Frankreich betr. Wehrdienst der Doppelstaater Inkrafttreten: 27. 12. 1934

Amtlicher Text: J. O. vom 4. 4. 1935, S. 3836

Französischer Text: State Papers Bd. 135, S. 467

Dalloz, B. L. 1935, S. 206

3. 6. 1929 Vertrag mit Peru über Tacna/Arica

StAB: Art. 10

Amtlicher Text: LNTS Bd. 94, S. 402

4. 6. 1956 Vertrag mit Italien über Wehrdienst der Doppelstaater

Inkrafttreten: 18. 10. 1959

Amtlicher Text: UNTS Bd. 362, S. 309 
24. 5. 1958 Vertrag mit Spanien betr. doppelte StA

Inkrafttreten: 28. 10. 1958. Dazu Notenwechsel v. 23.6. 58

Amtlicher Text: D. O. Nr. 24195 vom 15. 11. 58

(Dekret Nr. 569 vom 29.10.58)

Deutsche Übersetzung: SGS Bd. 11a, S. 31

Spanischer Text: SGS Bd. 11a, S. 31

Inf. Juridica 1959, S. 1345

13. 9. 1962 Vereinbarung mit Deutschland über Austausch von

Einbürgerungsmitteilungen

Amtlicher Text: B Anz. vom 19. 1. 63 (s. StAZ 1963, S. 150)

Neuere Literatur:

Garland, F. Toro

La doble nacionalidad. Comentario a la nueva ley chilena sobre la misma. in: Rev, de Derecho Español y Americano 1958, S. 339-347

Neue StA-Bestimmungen in Chile

in: StAZ 1959, S. 161

Hecker, $\mathrm{H}$.

Prieur-Kölling, W.

Miaja de la Muela, A.

Verfassungsrechts in Chile

in: JƠR, Bd. 16, S. 439-442 (StA)

El convenio hispano-chileno de doble nacionalidad, de 24. 5. 1958

in: Politica internacional 1960, Nr. 47, S. 85-108

Altere Literatur

Carmona de la Fuente, A.

Correa Bravo

Gurruchaga, A. O

Orrego Puelma, J.

Salas Troncoso

Examen critico de la nacionalidad . . Santiago 1925

in: Rev. de derecho jurispr. y letras (Buenos Aires), Bd. $33-$ Bd. 35

La nacionalidad y los modos de adquirirla, Santiago 1912

La nacionalidad, Santiago 1913

La nacionalidad originaria y adquirida, Santiago 1913

o. V.

Martens, N. R. G., 2. Serie, Bd. 19, S. $709-710$

\section{COLUMBIEN}

Übersicht über Rechtsquellen (1821-1955)

in: SGS Bd. 23, S. 42-47; Lit. S. 69-71

Literatur

Paula Perez, F. de

Derecho constitutional Columbiano

5. Auflage, Bogotá 1967, S. 147-161 (StA)

\section{COSTARICA}

Übersicht über Verfassungen (1825-1949), Gesetze (1841-1956),

Verträge (1850-1954) und Schrifttum

in: StAZ 1962, S. 195-196

\section{Gesetze:}

21. 11. (3. 12.) 1960 Gesetz Nr. 2701

ändert Art. 11 des Einbürgerungsgesetzes von 1950

Amtlicher Text: Gaceta Nr. 3 vom 5. 1. 1961

Spanischer Text: Colección de Leyes 1960 II, S. 497

9. (12.) 5. 1961 Gesetz Nr. 2739

ändert Art. 16 (StA-Verlust) der Verfassung von 1949

Amtlicher Text: Gaceta Nr. 111 vom 17. 5. 61

Spanischer Text: Colección 1961 I, S. 285

13. (28.) 8. 1965 Gesetz Nr. 3544

betr. Änderung von Art. 11 des Einbürgerungsgesetzes von 1950 unter Neuabdruck des ganzen Gesetzes

Amtlicher Text: Gaceta Nr. 198 vom 2. 9. 1965

$=$ Nr. 205 vom 10.9. 1965

Spanischer Text: BLE 1965, S. 319

Leyes usuales, San José 1966, S. 24; Colección 1965 II, S. 307 


\section{Verträge:}

8. 6. 1964 Doppelstaater-Konvention mit Spanien

Inkrafttreten: 21. 5. 1965

Amtlicher Text: BOE vom 25.6. 1965

Spanischer Text: EJE 1965, S. 893 (Instrument vom 21. 1. 65)

Literatur

Villegas Antillón, R.

Ley de extranjería y naturalización. Comentario y jurisprudencia. in: Revista del Colegio de Abogas 1962, Nr. 5, S. 41-84

9. CUBA

Vollständige Übersicht in StAZ 1969, S. 190-192

\section{DOMINIKANISCHE REPUBLIK}

\section{Verfassungen}

Sämtliche älteren Verfassungen sind abgedruckt in dem 1944 erschienenen zweibändigen Werk anläßlich der Hundertjahrfeier der Republik: Constitución politica, reformas constitucionales 1844-1942, Colección Trujillo, S. Domingo. Da die älteren Verfassungen vor 1881 meist nur dort abgedruckt sind, sei wegen der Fundstellen generell auf jenes Werk verwiesen. Genauere Angaben über alle Verfassungen siehe im Verfassungsregister der Forschungsstelle Teil III, S. 46.

1. 12. 1821 Verfassungsakt

StAB: Art. 9-12 u. 33

Englische Übersetzung: State Papers Bd. 8, S. 557

Spanischer Text: El Pensamiento Constitucional

18. 11. 1844 Verfassung

hispanoamericano hasta 1830, Caracas 1961, Bd. 4, S. 300

27. 2. 1854 Verfassung

StAB: Art. 5-6

23. 12. 1854 Verfassung

Englische Übersetzung: State Papers Bd. 46, S. 1315

19. 2.1858 Verfassung

außer Kraft: 27. 9. 1858

StAB: Art. 5-6

Englische Übersetzung: State Papers Bd. 48, S. 1052

14.11.1865 Verfassung

StAB: Art. 5-6

6. 9.1866 Verfassung

StAB: Art. 5-6, 10-11

Englische Übersetzung: State Papers Bd. 58, S. 333

23. 4. 1868 Verfassung

StAB: Art. 5

29.11.1872 Verfassung

StAB: Art. 5-6

4. 4. 1874 Verfassung

StAB: Art. 5-6

12. 4.1875 Verfassung

StAB: Art. 5-6

10. 5.1877 Verfassung

StAB: Art. 7-8 


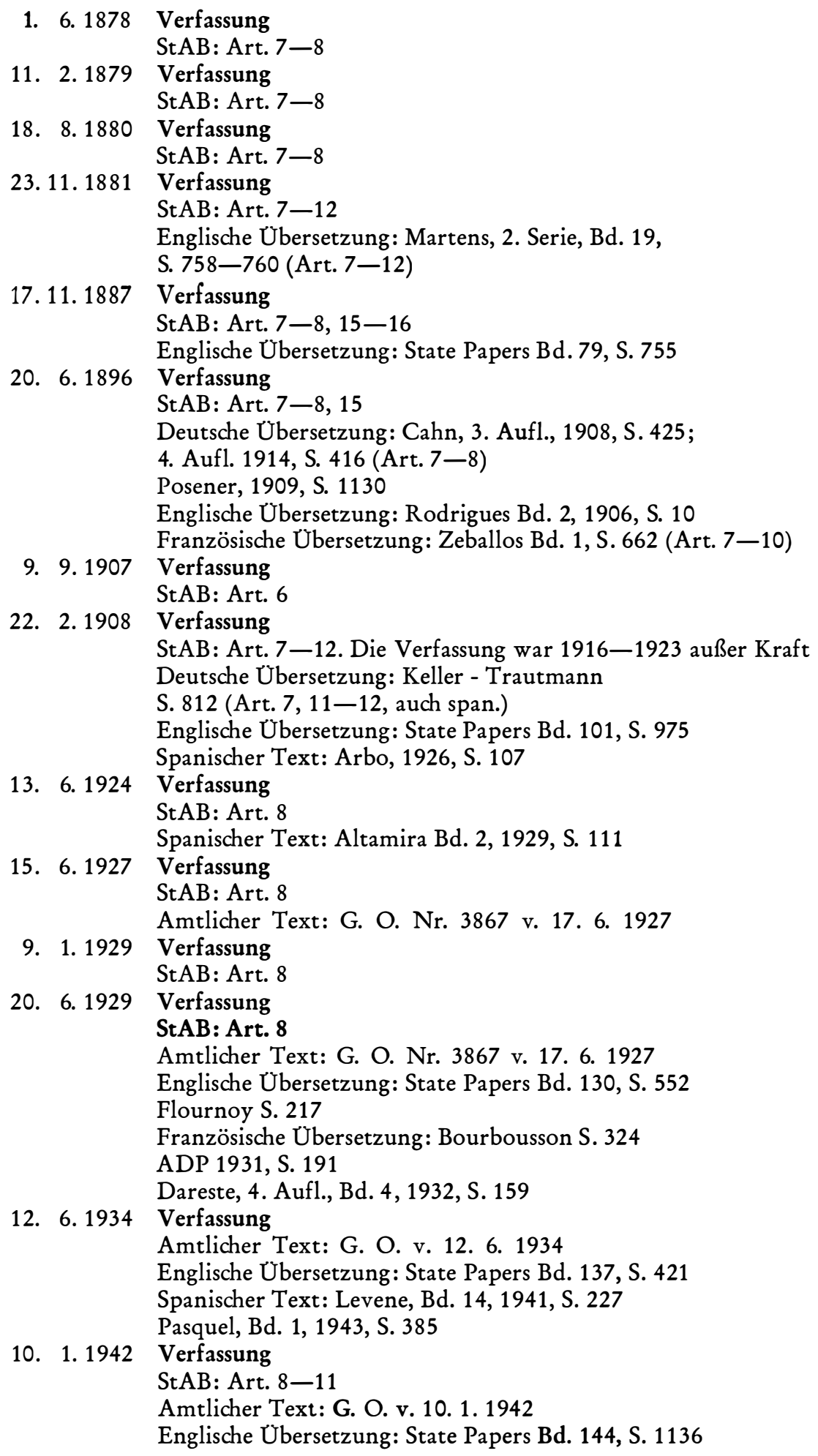


10. 1.1947 Verfassung

StAB: Art. 8

Amtlicher Text: G. O. Nr. 6569 v. 19. 1. 1947

Englische Úbersetzung: Peaslee, Bd. 1, S. 658 (1. Aufl.), 750 (2. Aufl.)

1. 12. 1955 Verfassung

Tellado, A statement . .., 1953, S. 1-22; LCN, S. 126 (nur Art. 8)

StAB: Art. $12-15$

Amtlicher Text: G. O. Nr. 7918 v. 2. 12.55

Englische Übersetzung: YHR 1955, S. 47

Tellado a. a. O., Suppl. 2, 1958, S. 1-27

Französische Übersetzung: NE Nr. 2612 v. 18. 12. 1959

Spanischer Text: Revista Dominica Nr. 54/55 v. 1955, S. 124

28. 6. 1960 Verfassung

StAB: Art. 12

Amtlicher Text: G. O. Nr. 8486 v. 29.6.60

Nr. 8527 bis v. 5.12 .60

Deutscher Text: Bergmann, 3. Aufl. (Art. 12)

Englischer Text: YHR 1960, S. 94

29. 12. 1961 Verfassung

Französischer Text: NE Nr. 2831 v. 10.11.61

StAB: Art. $12-15$

Amtlicher Text: G. O. Nr. 8631 v. 29.12. 1961

16. 9. 1962 Verfassung

StAB: Art. $12-15$

Amtlicher Text: G. O. Nr. 8693 v. 17. 9.62

Englischer Text: P. A. U., 1964, S. 1-27

29. 4. 1963 Verfassung

StAB: Art. 89-92

außer Kraft: 25. 11. 1963, mit welchem Tage wieder die Verfassung von 1962 in Kraft trat.

Amtlicher Text: G. O. Nr. 8758 v. 30. 4. 63

Spanischer Text: La Nacion v. 2. 5. 63, S. 10

28.11. 1966 Verfassung

StAB: Art. $11-15$

Amtlicher Text: G. O. Nr. 9014 v. 29. 11. 1966

\section{Gesetze}

21. 3. 1804 Codigo Civil (= Code Napoleon)

StAB: Art. 9-21. Erst 1884 wurde der Code, der noch unter französischer Herrschaft erlassen worden war, ins Spanische übersetzt, eine 2. Aufl. erschien in S. Domingo 1900 (Neudruck 1923). Die StA-Vorschriften des Code gelten noch heute, soweit nicht die Verfassungen und die Einbürgerungsgesetze (seit 1924) Abweichendes regeln. Ausdrücklich geändert wurden die StAB'en des Code durch Gesetze Nr. 485 v. 15. 1. 1944, Nr. 3354 v. 3. 8. 1952 und Nr. 3926 v. 18. 9.1954 (s. u.)

Englische Úbersetzung: LCN, 1954, S. 132 (nur Art. 12 und 19)

Spanischer Text: Codigo Civil, Santo Domingo 1930, S. 10,

Codigo Civil, Ciudad Trujillo 1950, S. 13

26. 9.1845 Dekret betr. Einbürgerung

erwähnt bei Cahn, 4. Aufl., S. 416 
18. 11. 1924 Erstes Einbürgerungsgesetz, Nr. 61

Amtlicher Text: O. G. Nr. 3595

4. 12. 1929 Zweites Einbürgerungsgesetz, Nr. 1227

In Art. 8 wird das erste Einbürgerungsgesetz aufgehoben.

Mit Änderungen und Ergänzungen v. 14. 11. 35, 1. 4. 36, 3. 2. 39, 15. 1. 44, 3. 8. 45, 5. 4. 46.

Englische Übersetzung: Flournoy, S. 218

Französische Übersetzung: Bourbousson, S. 323

1. 5. 1933 Gesetz Nr. 497 betr. Einbürgerungsgebühren

Amtlicher Text: G. O. Nr. 4572 v. 10. 5. 33, S. 3

14. 11.1935 Gesetz Nr. 1029 zur Ergänzung des Einbürgerungsgesetzes

Einziger Artikel: befreit einzubürgernde Soldaten von Abgaben

Amtlicher Text: G. O. Nov. 1935, S. 4

1. 4. 1936 Gesetz Nr. 1083 betr. bedingte Einbürgerung von Einwanderern

11 Artikel

Amtlicher Text: G. O. Nr. 4892 v. 4. 4.1936

3. 2. 1939 Gesetz Nr. 64 betr. Erleichterung der Einbürgerung von Soldaten

Einziger Artikel

Amtlicher Text: G. O. Febr. 1939, S. 5

6. 10. 1939 Gesetz Nr. 158 betr. Bedingungen der Einbürgerung

wegen besonderer Verdienste

Amtlicher Text: G. O. Nr. 5366 v. 7. 10. 1939

25. 7. 1941 Gesetz Nr. 508 betr. Einbürgerungsgebühren

7 Artikel

Amtlicher Text: G. O. Nr. 5618 v. 26. 7. 1941

30. 5. 1942 Gesetz Nr. 13 betr. Strafen für Dominikaner,

die eine ausländische StA beanspruchen

Amtlicher Text: G. O. Nr. 5757 v. 2. 6.1942

Englische Übersetzung: LCN, Nachtrag 1959, S. 36

(dort falsches Datum 3. 5)

4. 7. 1942 Gesetz Nr. 29 betr. Ersetzung des Gesetzes Nr. 13 von 1942

7 Artikel

Amtlicher Text: G. O. Nr. 5770 v. 8. 7. 1942

15. 1.1944 Gesetz Nr. 484 betr. Änderung von Art. 2 und 5

des Einbürgerungsgesetzes

3 Artikel

15. 1. 1944 Gesetz Nr. 485 betr. Änderung von Artikel 19 des CC

3. 8. 1945 Gesetz Nr. 961 betr. Einbürgerung wegen besonderer Verdienste

Ersetzt das Gesetz Nr. 158 v. 6. 10. 1939

Deutsche Übersetzung: Ex FfV

5. 4. 1946 Gesetz Nr. 1144 betr. Einbürgerungsgebühren

Amtlicher Text: G. O. Nr. 6424 v. 10. 4.1946

16. 4. 1948 Drittes Einbürgerungsgesetz, Nr. 1683

In Art. 33 wird das zweite Einbürgerungsgesetz aufgehoben.

Mit 7 Änderungen: 27. 8. 49, 16. 3. 1950, 10. 1. 51,

3. 8. $52,6.3 .55,16.9 .58,14.3 .60$ (s. u.)

Amtlicher Text: G. O. Nr. 6782 v. 1948

Deutsche Übersetzung: Bergmann, 3. Aufl., Dom. Rep. S. 2-6

(ohne Art. 27-33)

Englische Übersetzung: LCN, 1954, S. 126

Spanischer Text: Codigo Civil de la Rep. Dom., Ed.

Rodriguez, C. Trujillo 1950, S. 493-505

Colección de Leyes 1948, C. Trujillo 1950 
27. 8. 1949 Gesetz Nr. 2092 betr. Änderung von Art. 27

des Einbürgerungsgesetzes

Amtlicher Text: G. O. Nr. 6982, S. 8

16. 3. 1950 Gesetz Nr. 2303 betr. Änderung von Art. 27 III

des Einbürgerungsgesetzes

Amtlicher Text: G. O. Nr. 7095, S. 9

30. 12. 1950 Gesetz Nr. 2665 betr. Änderung von Art. 26

des Einbürgerungsgesetzes

Amtlicher Text: G. O. v. 10. 1. 1951

3. 8. 1952 Gesetz Nr. 3354 betr. Änderung von Art. 12 C. C.

3. 8. 1952 Gesetz Nr. 3355 betr. Anfügung eines $§$ III zu Art. 1

des Einbürgerungsgesetzes

18. 9. 1954 Gesetz Nr. 3926 betr. Anfügung eines zweiten Absatzes

zu Art. 19 C. C.

Amtlicher Text: G. O. Nr. 7747 v. 22. 9.1954

Englische Übersetzung: YHR 1954, S. 78

LCN, Nachtrag 1959, S. 36

6. 3. 1955 Gesetz Nr. 4063 betr. Änderung von Art. 1, 6 und 27

und Aufhebung von Art. 29 des Einbürgerungsgesetzes

Amtlicher Text: G. O. Nr. 7811 v. 9. 3. 1955

Englische Übersetzung: LCN, Nachtrag 1959, S. 34

Spanischer Text: Información jurídica 1956, S. 302 (Art. 1 und 6)

12. 9. 1956 Gesetz Nr. 4536 betr. Zurückziehung von Berufserlaubnissen

für Dominikaner, die eine fremde StA erwerben

Amtlicher Text: G. O. Nr. 8029 v. 22. 9.1956

Englische Übersetzung: LCN, Nachtrag 1959, S. 35

18. 9. 1958 Gesetz Nr. 4996 betr. Änderung von Art. 12II und

Art. 21 des Einbürgerungsgesetzes

Amtlicher Text: G. O. Nr. 8286 v. 20. 9. 1958, S. 8

11. 3. 1960 Gesetz Nr. 5322 betr. Anfügung von $₫ I-I I$ zu Art. 2

des Einbürgerungsgesetzes

Amtlicher Text: G. O. v. 14. 3. 1960

III. Verträge

Von multilateralen Verträgen trat die Dom. Rep. der Staatenlosen-Konvention von 1961, und zwar am 5. 9. 1961, bei sowie dem Wiener StA-Protokoll vom 18. 4. 1961 am 14. 1. 1964. Bilaterale StA-Verträge sind nicht bekannt, außer einem (s. u.). Die Annexion durch Spanien 1861-1865 und die Unterwerfung unter die USA 1916-1922 fanden keinen Niederschlag in StA-Bestimmungen. Ein Vertrag über die Angliederung an die USA von 1869 wurde zwar von S. Domingo, nicht aber von den USA ratifiziert: ob der Vertrag etwas über StA enthielt, war nicht festzustellen.

18. 2. 1855 Vertrag mit Spanien betr. Anerkennung der Unabhängigkeit,

Frieden, Freundschaft, Handel und Schiffahrt

StAB: entspricht Art. 9 des Vertrages mit Costarica v. 10. 5. 1850 betr. Unabhängigkeit. Der Vertrag war durch die Rückgliederung an Spanien (1861-1865) außer Kraft.

Französischer Text: State Papers Bd. 46, S. 1283

Literatur

Bourbousson, S. 66-68; Flournoy, S. 217; Lehr, S. 71-73

Tellado, A.

A statement of the laws of the Dom. Rep. in matters affecting business, Washington (P. A. U.) 2. Auflage 1953, S. 23-29

dazu Suppl. 1, 1956, S. 1-2; dazu Suppl. 2, 1958, S. 28-29

Planas Suárez, S

La nacionalidad. Ius soli. Legislatión dominicana, S. Domingo 1907

Zeballos, E.

La Nationalité

Bd. I, S. $662-664$, Bd. IV, S. $69 / 70,314-316,585$ f, $674 \mathrm{f}, 696 \mathrm{f}, 753 \mathrm{f}, 827 \mathrm{f}$

Fortsetzung im nächsten Heft 